\title{
Experimental Model of Pancreonecrosis Induced by Auto-bile Injection
}

\author{
Grigoriy Abramov*, Kairat Shakeyev, Maida Tusupbekova, Ermek Tagaev, Dmitriy Shestakov, Sanzhar Zhumanbaev \\ Karaganda Medical University, Karaganda, Kazakhstan
}

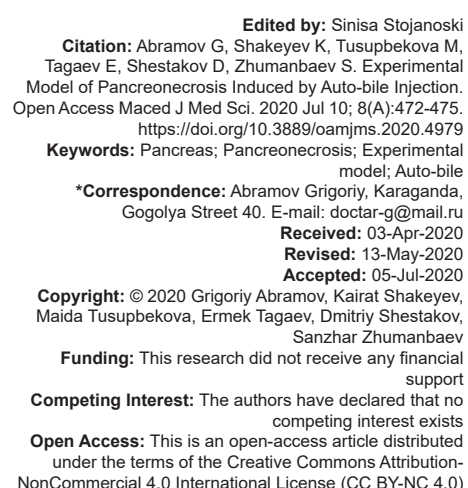

Edited by: Sinisa Stojanosk Edited by: Sinisa Stojanosk
Citation: Abramov G, Shakeyev K, Tusupbekova M Citation: Abramov G, Shakeyev K, Tusupbekova M Model of Pancreonecrosis Induced by Auto-bile Injection. Open Access Maced J Med Sci. 2020 Jul 10; 8(A):472-475. https://doi.org/10.3889/oamjms.2020.4979 Keywords: Pancreas; Pancreonecrosis; Experimental

*Correspondence: Abramov Grigoriy, Karaganda, Gogolya Street 40. E-mail: doctar-g@mail.ru Received: 03-Apr-2020 Revised: 13-May-2020 Copyright: ๑ 2020 Grigoriy Abramov, Kairat Shakeyev Maida Tusupbekova, Ermek Tagaev, Dmitriy Shestakov, Maida Tusupbekova, Ermek Tagaev, Dmitriy Shestakov, Funding: This research did not receive any financial Competing Interest: The authors have declared that no competing interest exists Open Access: This is an open-access article distributed under the terms of the Creative Commons Attribution-

\begin{abstract}
AIM: The aim of the study is to create an easily reproducible experimental model of pancreonecrosis with the least aggressive technique of the operation.

METHODS: Twenty-two outbred rabbits of comparable weight and age were included in the study. The animals were removed from the experiment 12,24 , and $48 \mathrm{~h}$ after the injection of auto-bile into the parenchyma of the pancreas.

RESULTS: After completion of the experiment, rabbit pancreas was extracted, macroscopic and microscopic description was given.

CONCLUSION: According to the results of the study, the proposed model is considered as consistent for reproducing pancreonecrosis in an experiment with less aggressive surgical technique.
\end{abstract}

\section{Introduction}

The method of injecting bile and its products into the biliopancreatic duct to reproduce pancreonecrosis is widely used in experimental surgery [1], [2]. The advantage of this method is the correspondence of the pathophysiological picture and the speed of development of necrosis. However, the significant disadvantage is the complexity of reproduction and considerable surgical aggression (opening of the duodenum and cannulation of the large papilla of the duodenum) that must be taken into account when processing the results.

\section{Materials and Methods}

The experiment was conducted on 22 outbred rabbits of both sexes weighing on average $2.5 \mathrm{~kg}$. Animal studies were conducted in accordance with international ethical requirements. Taking out of animals from the experiment was performed with anesthesia by euthanasia (exsanguination), that corresponds to GCP standards. To reproduce the model of pancreonecrosis, the abdominal cavity was opened under intravenous anesthesia with xylazine $0.2 \mathrm{mg} / \mathrm{kg}+$ ketamine $0.5 \mathrm{mg} /$ kg solutions (Figure 1a). Next, some bile was collected from the gallbladder with an insulin syringe (Figure 1b). Afterward, $0.1 \mathrm{ml}$ auto-bile was injected into the right and left pancreas lobe (Figure 1c) (note: A structural feature of the rabbit pancreas is the presence of two blades - the right and left ones) [3], [4], [5], [6]. Laparatomy wounds were sutured after an operation (Figure 1d). Taking out from the experiment was performed within $12(n=6)$, $24(n=6)$, and $48(n=6)$ hours after injecting auto-bile. Animals were being kept in standard conditions with free access to water and food. After that, the internal organs were taken for morphological studies. The entire pancreas was extracted; a macroscopic picture was described, and pancreas was fixed in $10 \%$ neutral formalin. Sections were stained with hematoxylin and eosin, examined by light microscopy. The results were compared with the results of the control group of intact animals $(n=4)$.

\section{Results}

The results of a pathomorphological study after the completion of the experiment showed that visually expressed structural changes in the rabbit pancreas according to macroscopic evaluation were not observed in the early stages (Figure 1). 


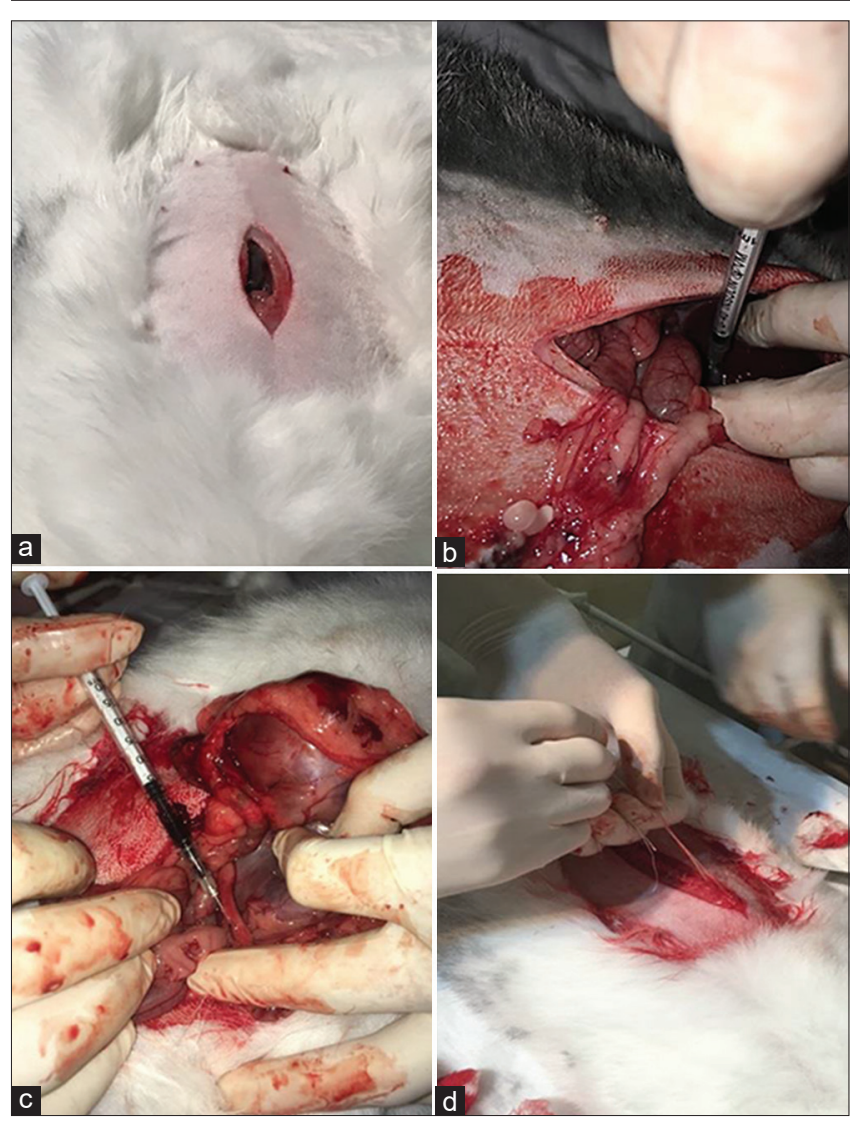

Figure 1: Operation procedure. Opening of the abdominal cavity, midmedian laparotomy (a); collecting auto-bile with an insulin syringe (b); subcapsular injection of auto-bile (c); suturing of a laparotomy wound (d)

At the completion of the experiment within 12 $\mathrm{h}$ after injecting auto-bile into the lobed lobes of the pancreas, macroscopic plethora of the capsule vessels and hemorrhages in the autologous injection zone was observed, the tissue was flabby elastic by consistency, the size of the organ did not undergo drastic changes, the length was $7.3 \mathrm{~cm}$, width $-1.8 \mathrm{~cm}$ (Figure 2a).

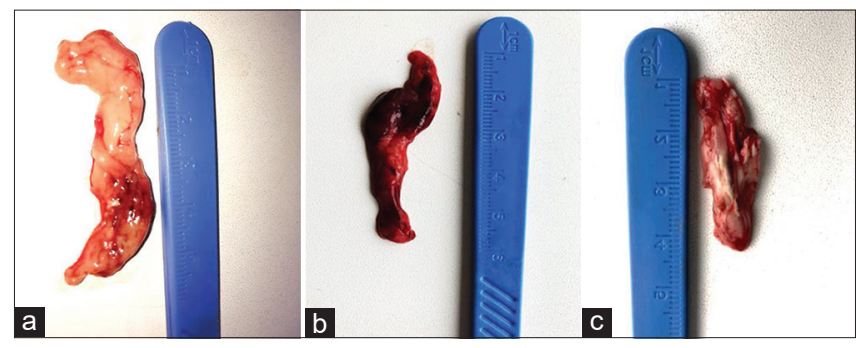

Figure 2: The preparation of rabbit pancreas (macroscopic appearance) terms of experience: (a) $12 \mathrm{~h}$ after injecting auto-bile, (b) $24 \mathrm{~h}$ after injecting auto-bile; (c) $48 \mathrm{~h}$ after injecting auto-bile

At the completion of the experiment within $24 \mathrm{~h}$ after injecting auto-bile, a decrease of the organ was macroscopically observed, its length was $5.5 \mathrm{~cm}$, width up to $1.0 \mathrm{~cm}$, focuses of merging hemorrhages were revealed, there was plethora of the pancreatic capsule vessels in the autologous administration zone, the parenchyma was flabby (Figure $2 b$ ).

At the completion of the experiment within $48 \mathrm{~h}$ after injecting auto-bile, visual studies showed that deformation and a sharp decrease of organ in size were observed macroscopically, length $3.7 \mathrm{~cm}$, width 1.0 and $0.8 \mathrm{~cm}$. Multiple foci of hemorrhage were found under the capsule, there was obvious plethora of vessels in the injection zone of auto-bile, the consistency is flabby (Figure 2c).

Anatomical and topographical and histological structure of human pancreas and experimental animals' (rabbits) are of the same type. As it was noted in a macroscopic study of the rabbit pancreas, it is covered with a thin membrane of loose fibrous connective tissue outside that divides the organ into lobules. The histological picture of the control group (intact) of pancreatic tissue is corresponded to the species norm.

In a histological examination, the exocrine part of the rabbit pancreas is represented by pancreatic lobules formed by numerous pancreatic acini with secretory sections; there are excretory ducts: Intercalated, intralobular, and interlobular ducts (Figure 3a). The interlobular space is represented by connective tissue fibers, where blood vessels and interlobular excretory ducts are found. The endocrine part is represented by round-shaped islets formed by endocrinocytes "Langerhans islets" (Figure 3b).

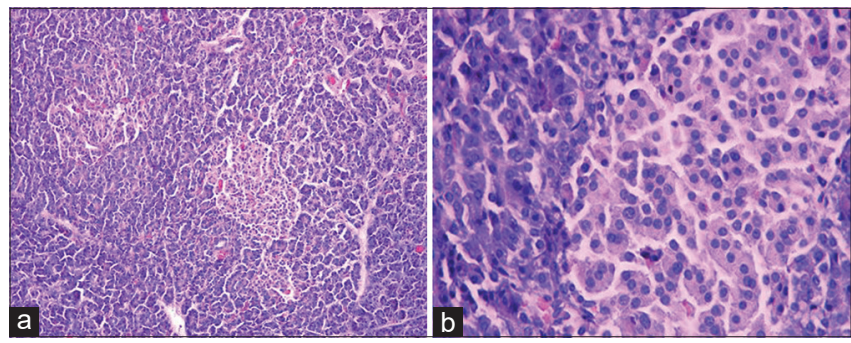

Figure 3: Rabbit pancreas (control): Pancreatic acini (Figure 1b) Langerhans islets. Coloring: Hematoxylin and eosin. Increased: (a) $\times 100$; (b) $\times 400$

Histological examination of the pancreas in terms of $12 \mathrm{~h}$ after the completion of the experiment, a structural disorder with the appearance of foci of necrobiotic changes with fuzzy acinar structures, pronounced interstitial edema (Figure 4a-c), vacuole dystrophy and parenchyma necrosis in the form of homogeneous blocky structures, or shadow outlines of the acini, foci, of hemorrhage were noted (Figure $4 \mathrm{~d}$ and e); foci of lipomatosis were detected with a preserved histostructure (Figure 4f).

A microscopic examination of the rabbit pancreas in terms of $24 \mathrm{~h}$ after injecting auto-bile histostructure disorders, significant interstitial edema, extensive foci of hemorrhage and diffuse lymphocytic leukocyte infiltration, and necrobiotic changes in the parenchyma are revealed, single acinar structures and ducts, extended and sanguineous vessels are observed (Figure 5).

The histological image of the pancreas in terms of $48 \mathrm{~h}$ after the experiment with injecting auto-bile; a violation of the histostructure with extensive areas of necrosis of exocrine acinar 


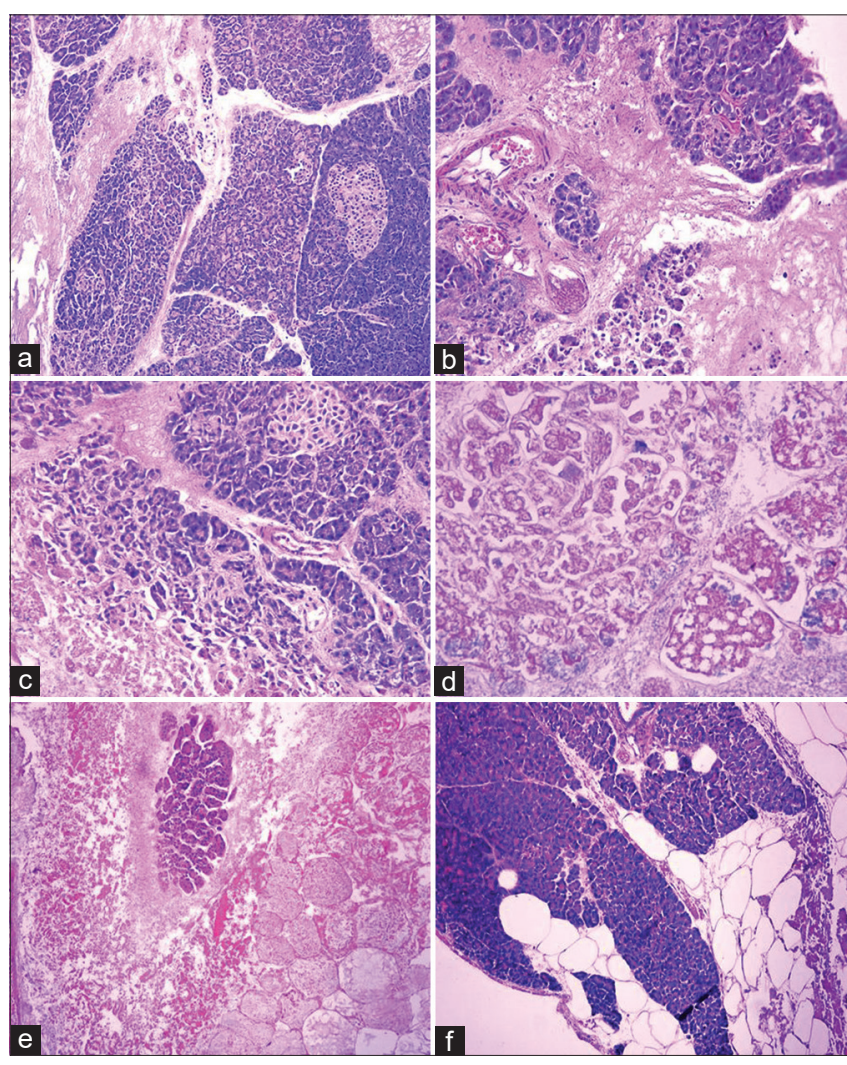

Figure 4: Pancreas, term - $12 \mathrm{~h}$. Focal necrobiotic changes, interstitial edema (a-c), necrosis of acinar exocrinocytes, foci of hemorrhage ( $d$ and e), foci of lipomatosis (f). Coloring: Hematoxylin and eosin. Increased: $\times 100$

structures and ducts, parenchyma necrosis foci in the form of homogeneous, and nuclear shapes are noted (Figure 6a-d), in other areas - fatty vacuole dystrophy and foci of lipomatosis are observed, Figure $6 \mathrm{e}$ and $\mathrm{f}$.

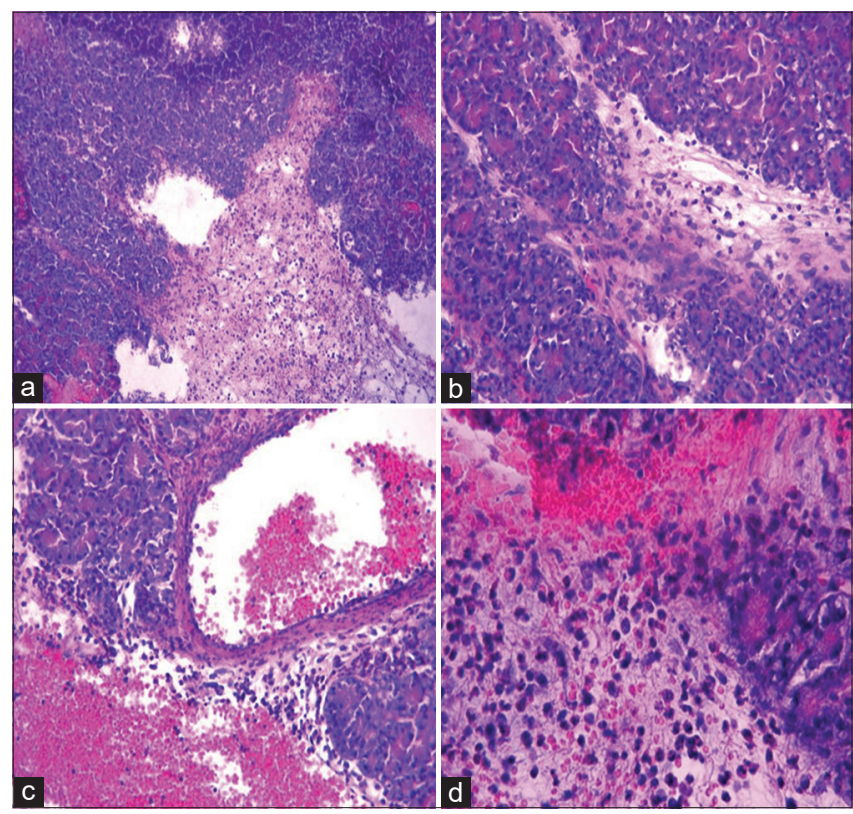

Figure 5: Pancreas, term - $24 \mathrm{~h}$. Disruption of the histostructure: Necrobiotic changes in acinar structures, interstitial edema with diffuse lymphocytic leukocyte infiltration, foci of hemorrhage, and vascular congestion (a-d) Coloring: Hematoxylin and eosin Increased: $(a$ and $b) \times 100$, $(c$ and d $) \times 400$, $(d$ and e) $\times 200$
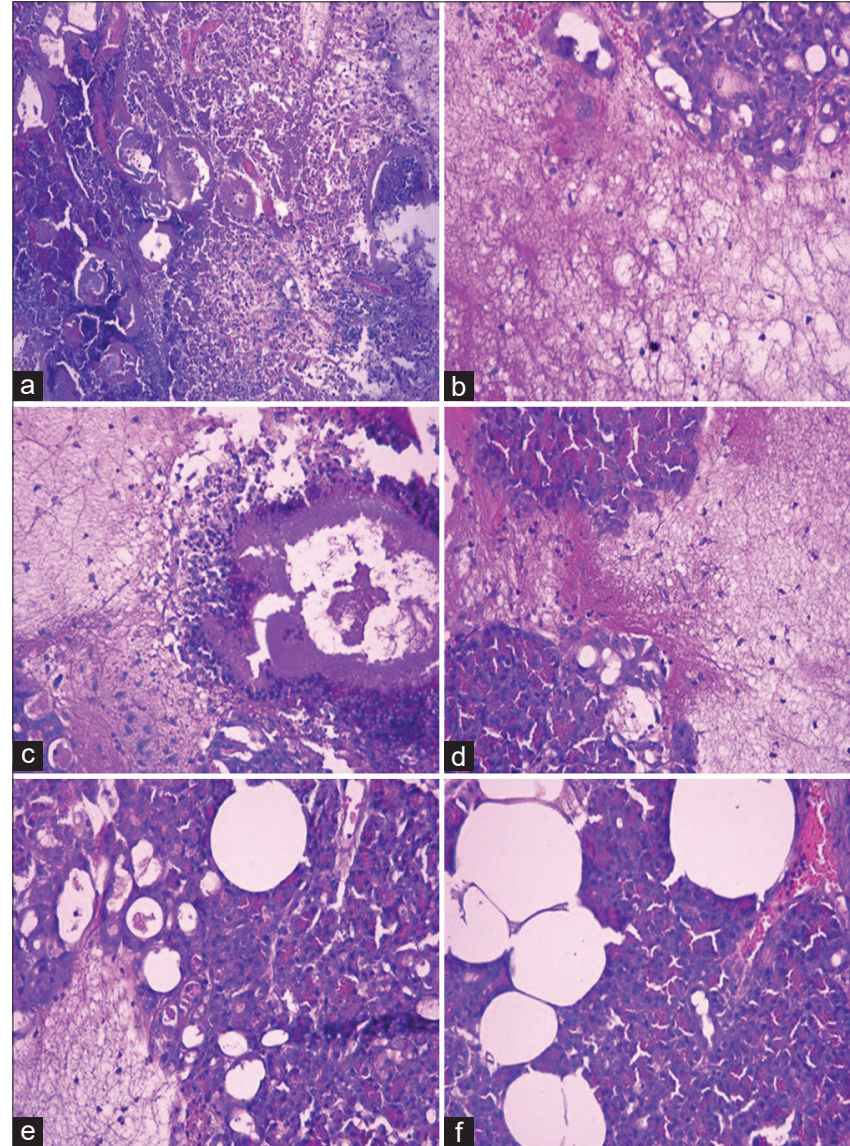

Figure 6: Pancreas, term - $48 \mathrm{~h}$. Violation of the structure with extensive areas of necrosis (a-d), lipomatosis foci (e and f). Coloring: Hematoxylin and eosin. Increased: $(a$ and b) $\times 100,(c-f) \times 200$

\section{Discussion}

The results of a pathomorphological study showed that visually expressed structural changes in the rabbit pancreas in the early stages by means of macroscopic evaluation were not observed. At the term of $12 \mathrm{~h}$ after completion of the experiment, plethora of the capsule vessels and hemorrhages foci in the zone of auto-bile injection was macroscopically observed. The size of the organ did not change drastically. Histology was characterized by the emergence of foci necrobiotic changes. At the term of $24 \mathrm{~h}$ after the completion of the experiment, pancreas decreased in size. It was noted histologically that organ histostructure was disordered. Diffuse lymphocytic leukocyte infiltration and necrobiotic changes in parenchyma were observed. At the term of $48 \mathrm{~h}$ after the completion of the experiment, it was observed macroscopically that pancreas sharply decreased in size, multiple foci of hemorrhage appeared, extensive areas of exocrine apparatus necrosis and foci of parenchyma necrosis in the form of homogeneous, nuclear shapes were histologically noted. Thus, this model is regarded as consistent for reproducing pancreatic necrosis in an experiment with less aggressive surgical technique. 


\section{Conclusion}

An experimental model of pancreatic necrosis induced by the administration of auto-bile is regarded as consistent and easily reproducible. The creation of this model is also carried out with a less aggressive operational technique, which, in turn, affects biochemical parameters to a lesser extent.

\section{References}

1. Abramov GA, Shakeev KT, Tusupbekova MM, Tagaev EE, Shestakov DV, Zhumanbaev SM. Modeling pancreatic necrosis in an experiment. Certificate on state registration of rights to the subject of copyright No. 71902019 dated 12/23/2019, Kazakhstan.

2. Agapov MA, Gorsky VA, Petrov VA, Polivoda MD, Kravchenko AYu, Battaev Al. Methods of modeling acute pancreatitis (literature review). Bull Russ State Med Univ 2014;3:25-9.

3. Gushchin YI, Shedko VV, Muzhikyan AA, Makarov MN, Makarov VG. Comparative morphology of the pancreas of experimental animals and humans. Lab Anim Sci Res 2018;3:33-48.

4. Kirillovyh AS. Morphometric characteristics of the endocrine part of the rodent pancreas in acute destructive pancreatitis. BBC 2011;30:38.

5. Makarov MN, Rybakova AB, Gushchin YA, Shedko BB, Muzhikyan AA, Makarov VG. Anatomical and physiological characteristics of the digestive tract of humans and laboratory animals. Int Bull Vet Med 2016;1:82-104.

6. Petrenko VM. The shape and topography of the pancreas. Int $\mathrm{J}$ Appl Basic Res 2015;1:1114-7. 